\title{
Oblique interlaminar lumbar epidural steroid injection for management of low back pain with lumbosacral radicular pain - A case report -
}

Received February 22, 2017

Revised 1st, May 1, 2017

2nd, May 11, 2017

Accepted May 11, 2017

\section{Corresponding author}

Eun-Ji Choi, M.D.

Department of Anesthesia and Pain Medicine, Pusan National University School of Medicine, 20, Geumoro, Mulgeum-eup, Yangsan 50612, Korea

Tel: 82-55-360-2758

Fax: 82-55-360-2149

E-mail: eunjichoi81@gmail.com

\section{Gyeong-Jo Byeon, Eun-Ji Choi, Yun-Mi Choi, Eun-Jung Chang, Hye-Jin Kim, and Kyung-Hoon Kim}

\author{
Department of Anesthesia and Pain Medicine, Pusan National University School of
} Medicine, Yangsan, Korea

\begin{abstract}
Epidural steroid injection (ESI), which is commonly used for treatment of low back pain with lumbosacral radicular pain, can be performed via transforaminal, interlaminar, or caudal routes. The transforaminal route is generally regarded as more effective than the interlaminar route due to its high level of drug delivery to the ventral epidural space. However, in some postoperative patients, use of the transforaminal route may be difficult. Thus, there is an urgent need for technology that can offer more effective drug delivery to the ventral epidural space with fewer complications. In this context, we describe a case about our new method where patient has undergone oblique interlaminar lumbar epidural steroid injection (OIL-ESI) instead of transforaminal ESI. We treated a patient with OIL-ESI instead of transforaminal ESI. Patient was symptomatic improved at postoperative visits. Based on our findings, OIL-ESI may be a suitable alternative to transforaminal ESI.
\end{abstract}

Key Words: Epidural injection, Fluoroscopy, Low back pain, Pain management, Steroid.
Epidural steroid injection (ESI) is an effective treatment method for patients with low back pain and lumbosacral radiating pain. It relieves symptoms by injecting anti-inflammatory medication into the perineural environment of the affected nerve root. It can be performed via transforaminal, interlaminar, or caudal approaches. Transforaminal ESI is widely used as it has low risk of dural puncture and can effectively deliver a relatively small amount of the drug to inflamed sites via the ventral epidural space [1]. However, in some postoperative states, the transforaminal route may be difficult due to the presence of rods, pedicle screws, and bone harvests for posterior lumbar interbody fusion. Sometimes, serious complications, such as spinal cord injury and spinal infarction, have also been reported [2]. For these reasons, various treatment methods have been proposed that offer reduced complications, are safe, and have comparable efficacy to that of transforaminal ESI [3-5].

In this report, we describe our successful experience using oblique interlaminar lumbar epidural steroid injection (OILESI) to treat patient with low back pain and lumbosacral radicular pain, and a literature review.

\section{CASE REPORT}

An 82-year-old female patient who had undergone posterior lumbar interbody fusion in the L4-L5-S1 region eighteen years ago was admitted to our pain clinic. She complained of pain in the lower back and left lower extremity associated

This is an Open Access article distributed under the terms of the Creative Commons Attribution Non-Commercial License (http://creativecommons.org/licenses/by-nc/4.0) which permits unrestricted non-commercial use, distribution, and reproduction in any medium, provided the original work is properly cited. 
with left lower extremity radicular pain and paresthesia in the L5 dermatome. The patient also complained of gait disturbance and intermittent claudication and her numeric rating scale (NRS) score for pain was 9/10. The physical examination revealed positive straight leg raise test results in the left lower extremity and tenderness in the third, fourth, and fifth lumbar transverse process areas.

Prior to her admission to our clinic, the patient underwent medial branch block and caudal epidural block at a local clinic; however, since her symptoms did not improve, facet joint syndrome due to postoperative junctional problems was excluded from the diagnosis. A lumbar spine computer tomography (CT) was obtained, the results of which she presented to us at the time of her admission. Although the CT did not show any distinct nerve compression caused by the screws, the patient was exhibiting symptoms of neuropathy, such as severe leg numbness.

Initially, we planned to administer a caudal epidural block with associated oral medication. With the patient in prone position, and after local infiltration with lidocaine $1 \%$ at the sacral hiatus, a 22-G Tuohy needle (Hakko, Nagano, Japan) was inserted into the sacral canal. Once the needle reached the ventral wall of the sacral canal, $3 \mathrm{ml}$ of iopamidol was injected. When we observed the dispersion of contrast into the epidural space in real-time fluoroscopic anteroposterior (AP) imaging, the contrast was not dispersed up to the S1 level (Fig. 1). After confirming negative dispersion of contrast into vessels, dexamethasone $5 \mathrm{mg}$ and hyaluronidase 1,500 IU, mixed with $20 \mathrm{ml}$ of lidocaine $1 \%$, was injected into the epidural space.
At the patient's clinic appointment 1 week later, she stated that her left lower extremity pain and paresthesia on L5 dermatome did not improve. Therefore, we planned to administer transforaminal ESI at the L4-L5 level. The patient was given full knowledge of the risks involved, including radiation exposure, probable adverse effects, and the alternatives, and subsequently provided consent. An initial AP fluoroscopic image was obtained to identify the level of the intervertebral disc and interlaminar space with the patient in a prone position and a pillow placed under his abdomen.

Under sterile conditions and after local infiltration with lidocaine 1\%, a 22-G, 100-mm Quincke needle (Hakko Co. Ltd, Japan) was inserted at the level of disc pathology using the AP view and then an oblique orientation of the fluoroscopy to achieve the 'Scotty Dog' appearance of the lumbar spine. It was then directed until the needle tip was in the posterior and superior aspect of the intervertebral foramen, as reviewed on lateral imaging, and in line with the pedicle on AP view. However, the needle was not advanced into the intervertebral foramen due to rods, pedicle screws, and bone harvests for posterior lumbar interbody fusion. Therefore, we switched to the oblique interlaminar route for ESI (Fig. 2). After the patient gave informed consent and under sterile conditions and after local infiltration with lidocaine 1\%, a 22 G, 80-mm Tuohy needle (Hakko Co. Ltd, Japan) was inserted at the location of disc pathology (L4-L5 interlaminar space). For the oblique interlaminar approach, fluoroscopy was oriented right obliquely 15 degrees in the caudocephalad direction to achieve the 'Scotty Dog' appearance. The needle was advanced in a slight cephalad direction toward the left
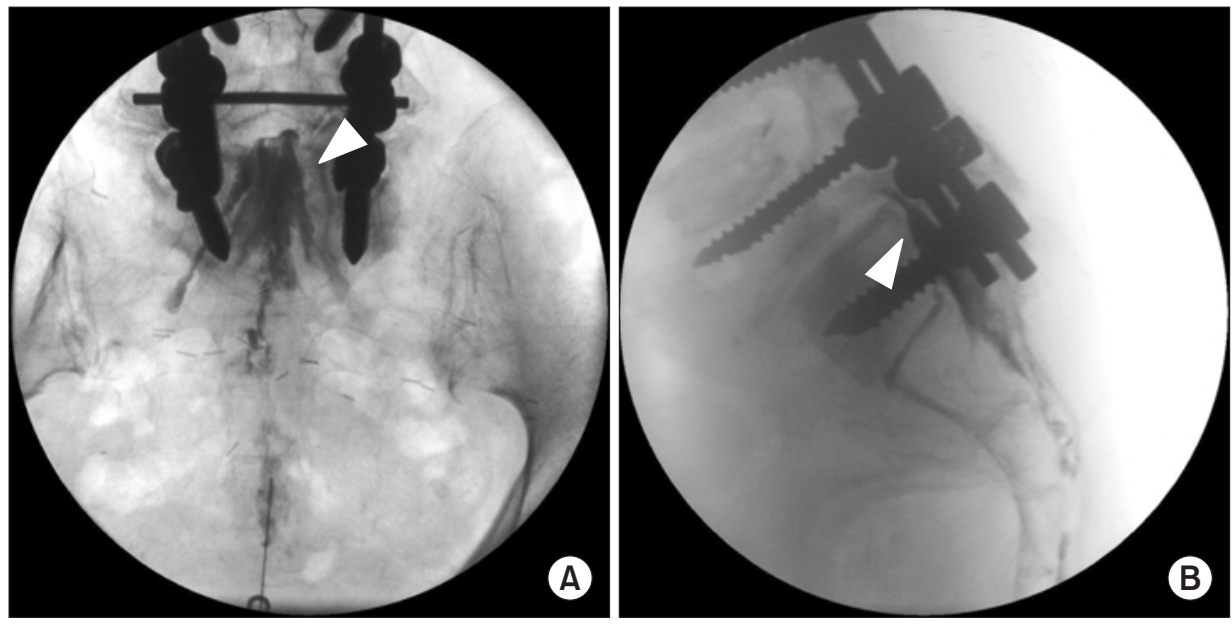

Fig. 1. Fluoroscopic images of contrast spread after caudal epidural block. (A) Anteroposterior view of contrast spread. (B) Lateral view of contrast spread. Arrowhead: contrast spread in the epidural space. 

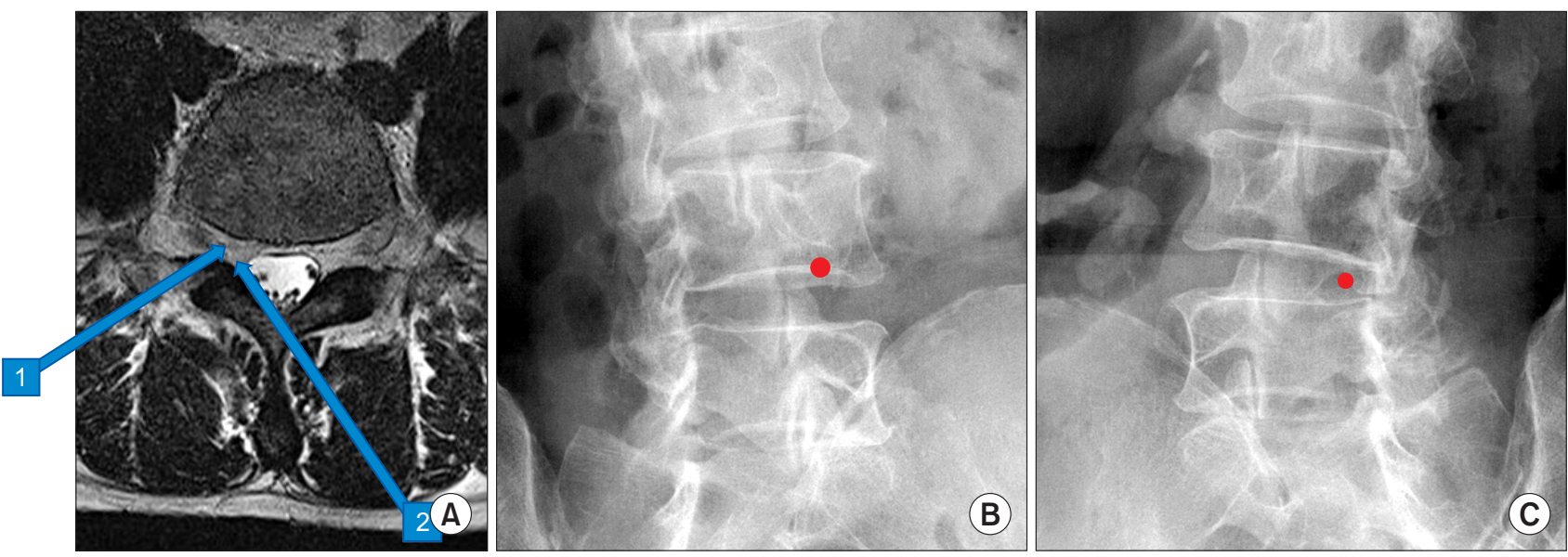

1. Transforaminal approach

2. Oblique interlaminar approach

Fig. 2. Comparison of transforaminal versus oblique interlaminar approach. (A) Needle entry points of transforaminal approach versus oblique interlaminar approach. (B) Initial needle entry point of right transforaminal approach. (C) Initial needle entry point of right oblique interlaminar approach.
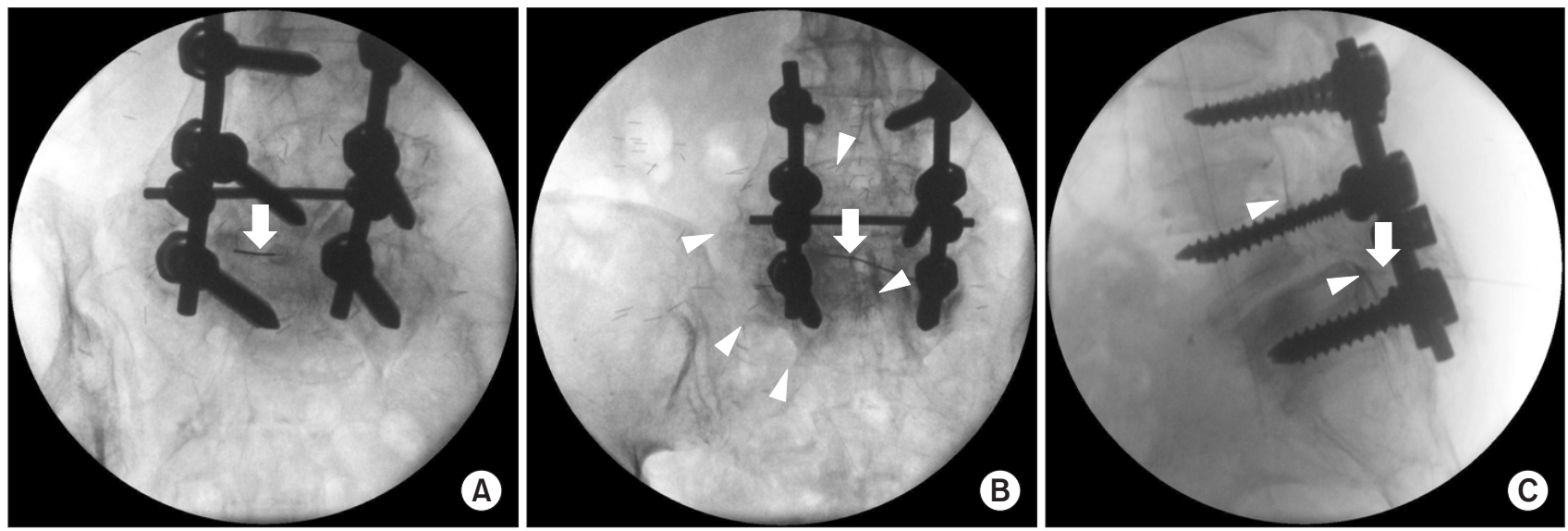

Fig. 3. Fluoroscopic images of contrast spread after oblique interlaminar approach. (A) Initial entry point of right oblique L5-S1 interlaminar approach. (B) Anteroposterior view of contrast spread. (C) Lateral view of contrast spread. Arrow: a 22-G, 80-mm Tuohy needle, Arrowhead: contrast spread in the epidural space.

most lateral part of the interlaminar opening, as indicated by the right oblique projection on fluoroscopy. The needle was inserted into the left side of the epidural space using the loss of resistance to saline technique and the oblique orientation of the needle was maintained throughout the procedure. Once the needle was in position, and after negative aspiration for cerebrospinal fluid and blood, iopamidol (Pamiray 300; Dongkook Pharm. Co. Ltd., Korea) was injected using real-time, continuous fluoroscopy for the entire $3 \mathrm{ml}$ volume of injectate and images were obtained in the lateral and AP projections (Fig. 3). This was monitored to confirm spread of contrast and to verify that no contrast medium entered the intravascular, subarachnoid, subdural, or intradiscal spaces. Lateral images were taken to evaluate the ventral epidural space. Perineural and segmental spread were also visualized in the AP view. After epidural space confirmation, dexamethasone $5 \mathrm{mg}$ and hyaluronidase 1,500 IU, mixed with 4 $\mathrm{ml}$ lidocaine $1 \%$, was injected into the epidural space.

The patient stayed at the day clinic for $30 \mathrm{~min}$ for observation and she was discharged without any neurologic complications. When she visited the clinic 1 week after the procedure, there was still slight radiating pain (NRS score $=5 / 10$ pain). Two weeks later, the patient received an additional OIL-ESI. After 1 month, the patient reported only slight pain 
(NRS score $=3 / 10$ pain $).$

\section{DISCUSSION}

Epidural steroid injection is an effective treatment method for patients with low back pain who do not respond to conservative treatments [1]. The mechanism of low back pain with radiculopathy was originally thought to be associated with direct pressure on nerve roots caused by herniated discs and/or spinal stenosis. However, currently inflammatory response and various inflammatory events, in addition to mechanical pressure, have been reported as the major causes of radiculopathy $[6,7]$. A previous study reported that phospholipase A2 activity at the site of lumbar herniated intervertebral discs increased 100,000-fold compared with that in other tissues [8]. Phospholipase A2 liberates arachidonic acid from cell membranes, which produces prostaglandin and leukotriene inflammatory mediators [1]. Steroids inhibit this inflammatory response by inhibiting phospholipase A2 [9] . Meanwhile, the treatment result of hyaluronidase on postspinal surgery syndrome patients with pain, which is caused by epidural fibrosis due to dissolving of the connective tissues, has been reported. Rahimzadeh et al. [10] reported that adding 1,500 IU of hyaluronidase to epidural steroid injection proved to be effective for post-spinal surgery syndrome patients. Moreover, although few studies have reported allergic reactions during epidural injection of hyaluronidase, such reactions were rare and not fatal. Consequently, it was used in this case based on our determination that hyaluronidase could improve the dispersion of the medication by reducing tissue swelling $[10,11]$.

The effectiveness of ESI treatment depends on steroids reaching the nerve root lesion. Most patho-physiologies exist in the ventral epidural space where interactions between discs and the dura mater occur. Thus, the primary goal of ESI for low back and radiating leg pain is ensuring that the injected drugs reach the ventral epidural space and dorsal root ganglion [12,13]. There are differences among these 3 approaches. The interlaminar route is safe, easy to use, and requires smaller amounts of the drug than dose the caudal route. The transforaminal route is target-specific; hence, it uses the least amount of the drug. The caudal route is safe and the easiest to perform among the 3 routes, but requires the largest amount of the drug to reach the lesion [1].
Transforaminal ESI is the favored method for approaching the ventral epidural space. It has the highest potential for delivering the drug to the ventral epidural space compared with interlaminar and caudal epidural steroid injections. A previous study reported that only $36 \%$ of the drug injected via interlaminar ESI reached the ventral epidural space [14]. Transforaminal ESI offers many advantages over the interlaminar route such as reduced risk of dural puncture, injection of the drug closer to the nerve root where the lesion is located, delivery of the drug to the ventral epidural space, and less drug required compared with other routes.

In addition to mild complications following transforaminal ESI, such as temporary increase in low back pain, headache and blood sugar elevation [15], serious complications (e.g., spinal cord infarction) have also been reported [2]. Relative to the number of times that transforaminal ESI is performed, such complications are rare but cannot be overlooked due to their potentially serious consequences. Based on reports of these serious complications, the efficacy, safety, and approaches of this treatment should be re-examined and new treatment methods developed to address these concerns.

There are few studies that have attempted ventral epidural ESI into the interlaminar epidural space. Previous one study divided patients with low back pain into parasagittal and transforaminal ESI treatment groups, and reported spread of contrast media into the ventral epidural space in $100 \%$ and $75 \%$ patients who had undergone interlaminar parasagittal and transforaminal approaches, respectively [3]. The other study compared the results of positioning the catheter tip on the ventral or dorsal spinal nerve via retrograde interlaminar ventral epidural injections and reported no difference between the two groups [4]. Another study performed epidural perineural injection for treating lumbar radicular pain and reported that the procedure was effective [5]. Basically, epidural perineural injection has the same needle approach as OIL, OIL approach found a needle entry point using fluoroscopy and this is an advantage of the OIL approach. The OILESI allows fluoroscopic guided needle approach by identifying the needle entry point, which it is more precise procedure than epidural perineural injection.

In the presented case, OIL-ESI was performed on a patient with low back pain accompanied by lumbosacral radicular pain. Following treatment, the patient reported significant reduction in NRS scores. In the case, the transforaminal ap- 
proach was attempted since the initial caudal approach was not effective. However, the procedure was difficult due to the history of surgery. Where the patient had a surgical history, selective nerve root block using the spring-guide catheter (Racz catheter) or injection with epiduroscopy may have been a good alternative. However, neither method was opted for as the first choice due to relatively high cost. In addition, because the patient was in extreme pain at the time, pain management was critical. Consequently, we performed the OIL approach despite the risk of dural puncture or infection. In post-spinal-surgery patients, the OIL approach should be carefully assessed before selection of this method. This useful approach can be employed when the transforaminal approach is difficult to perform due to anatomical deformity.

While transforaminal ESI is simple and easy to perform manually, placement of the nerve block needle may be difficult due to abnormalities in the intervertebral foramen caused by intervertebral foraminal stenosis, spondylolisthesis, scoliosis, or surgical history. Therefore, OIL-ESI should be considered in cases where the transforaminal route may not be feasible due to anatomic deformation in the lumbar region.

OIL approach is a part of the interlaminar approach and has a lower risk of spinal cord infarction compared to the transforaminal approach; however, there is a risk of dural puncture and nerve injury. The OIL method uses an approach from the opposite direction, and thus, the possibility of dural puncture and nerve root injury could not be dismissed. However, the loss of resistance to saline technique was used while checking the epidural needle depth via fluoroscopic lateral view to reduce the risk. In the OIL approach, the needle is inserted more laterally and obliquely than in the traditional interlaminar approach, and as a result, drugs are expected to be delivered more readily into the ventral epidural space than by the traditional approach. Currently, there are many on-going studies that are collecting cases for comparative studies between the OIL and interlaminar approaches. The OIL technique may be slightly more difficult than the traditional interlaminar approach. However, since this method is indicated when transforaminal ESI is difficult, the OIL technique offers enough advantages for consideration.

In post-spinal-surgery patients, the OIL approach should be carefully assessed before selection of this method. This useful approach can be employed when the transforaminal approach is difficult to perform due to anatomical deformity. OIL is a modified interlaminar approach, and therefore, the adverse event profile is also predicted to be similar to that of the interlaminar approach. However, large-scale clinical studies are required as this approach is a relatively new method.

In conclusion, OIL-ESI is a good alternative that shows efficacy similar to the transforaminal approach by delivering medications to the ventral epidural space and can be used instead of transforaminal ESI in patients with lower back pain concerned about severe adverse events (e.g., spinal cord infarction) or when transforaminal ESI is difficult to perform due to anatomical deformities.

\section{ACKNOWLEDGMENTS}

This work was supported by clinical research grant from Pusan National University Yangsan Hospital in 2016.

\section{REFERENCES}

1. Abdi S, Datta S, Lucas LF. Role of epidural steroids in the management of chronic spinal pain: a systematic review of effectiveness and complications. Pain Physician 2005; 8: 127-43.

2. Quintero N, Laffont I, Bouhmidi L, Rech C, Schneider AE, Gavardin $\mathrm{T}$, et al. Transforaminal epidural steroid injection and paraplegia: case report and bibliographic review. Ann Readapt Med Phys 2006; 49: 242-7.

3. Candido KD, Raghavendra MS, Chinthagada M, Badiee S, Trepashko DW. A prospective evaluation of iodinated contrast flow patterns with fluoroscopically guided lumbar epidural steroid injections: the lateral parasagittal interlaminar epidural approach versus the transforaminal epidural approach. Anesth Analg 2008; 106: 638-44.

4. Jeong JS, Shim JC, Woo JP, Shim JH, Kim DW, Kim KS. Epidural contrast flow patterns of retrograde interlaminar ventral epidural injections stratified by the final catheter tip placement. Anesth Pain Med 2013; 8: 158-65.

5. Kraemer J, Ludwig J, Bickert U, Owczarek V, Traupe M. Lumbar epidural perineural injection: a new technique. Eur Spine J 1997; 6: 357-61.

6. Sizer PS Jr, Phelps V, Dedrick G, Matthijs O. Differential diagnosis and management of spinal nerve root-related pain. Pain Pract 2002; 2: 98-121.

7. Cavanaugh JM. Neural mechanisms of lumbar pain. Spine (Phila 
Pa 1976) 1995; 20: 1804-9.

8. Saal JS, Franson RC, Dobrow R, Saal JA, White AH, Goldthwaite N. High levels of inflammatory phospholipase A2 activity in lumbar disc herniations. Spine (Phila Pa 1976) 1990; 15: 674-8.

9. Lee HM, Weinstein JN, Meller ST, Hayashi N, Spratt KF, Gebhart GF. The role of steroids and their effects on phospholipase A2. An animal model of radiculopathy. Spine (Phila Pa 1976) 1998; 23: 1191-6.

10. Rahimzadeh P, Sharma V, Imani F, Faiz HR, Ghodraty MR, Nikzad-Jamnani AR, et al. Adjuvant hyaluronidase to epidural steroid improves the quality of analgesia in failed back surgery syndrome: a prospective randomized clinical trial. Pain Physician 2014; 17: E75-82.

11. Park AR, Kim WM, Heo BH. Delayed allergic reaction to secondary administrated epidural hyaluronidase. Korean J Pain 2015;
28: $153-5$.

12. Lutz GE, Vad VB, Wisneski RJ. Fluoroscopic transforaminal lumbar epidural steroids: an outcome study. Arch Phys Med Rehabil 1998; 79: 1362-6.

13. Manchikanti L. Transforaminal lumbar epidural steroid injections. Pain Physician 2000; 3: 374-98.

14. Botwin KP, Natalicchio J, Hanna A. Fluoroscopic guided lumbar interlaminar epidural injections: a prospective evaluation of epidurography contrast patterns and anatomical review of the epidural space. Pain Physician 2004; 7: 77-80.

15. Botwin KP, Gruber RD, Bouchlas CG, Torres-Ramos FM, Freeman TL, Slaten WK. Complications of fluoroscopically guided transforaminal lumbar epidural injections. Arch Phys Med Rehabil 2000; 81: 1045-50. 